\title{
Impacto de la exposición prenatal a testosterona sobre parámetros biométricos y endocrinos en ovinos recién nacidos ${ }^{\#}$
}

\author{
Impact of prenatal exposure to testosterone in biometrics and endocrine parameters \\ of newborn lambs \\ SE Recabarren ${ }^{a^{*}}$, PP Rojas-García ${ }^{a}$, MP Recabarren ${ }^{a}$, K Norambuena $^{a}$, T Sir-Petermann ${ }^{b}$ \\ aLaboratorio de Fisiología y Endocrinología Animal, Facultad de Ciencias Veterinarias, Universidad de Concepción, Chillán, Chile. \\ bLaboratorio de Endocrinología y Metabolismo, Facultad de Medicina Occidente, Universidad de Chile, Santiago, Chile.
}

\begin{abstract}
SUMMARY
Biometric and endocrine parameters were studied in male and female Suffolk Down newborn lambs born to mothers exposed to an excess of testosterone (EPT) during 60 days (EPT-P1) and during 90 days (EPT-P2), beginning at day 30 of pregnancy. Control mothers received vegetable oil. At delivery time the following data were registered: time of delivery, number of newborns per mother and sex of the newborn. At 8 hours postpartum, the characteristics of the genitalia, the anus-urinary meatus distance and body weight were recorded. Blood samples were taken to measure cortisol, $\mathrm{T} 3$ and T4 on plasma. Data regarding time of birth, number of offspring and sex did not show any differences between the three groups of newborn lambs. Females born to EPT-P1 and EPT-P2 mothers exhibited masculinized genitalia, showing a penis and an empty scrotum. Females born to EPT-P1 mothers showed lower body weight and lower plasma T4 concentrations than females and males born to the other 2 groups of mothers $(\mathrm{P}<0.05)$. Plasma cortisol concentrations were not different between offspring from the 3 groups of mothers, however, females born to EPT-P1 mothers showed an inverse correlation between cortisol concentration and body weight. Data showed that exposure to testosterone during a critical time during pregnancy had a profound impact on genitalia of females, and depending on the dose it produces lower body weight and lower plasma T4 concentrations in newborn females. The EPT had no effect on biometric and endocrine parameters in newborn male lambs.
\end{abstract}

Palabras clave: testosterona, masculinización, hormonas tiroideas, programación fetal.

Key words: testosterone, masculinization of external genitalia, thyroid hormones, fetal programming.

\section{INTRODUCCIÓN}

La oveja constituye un valioso modelo experimental para el estudio de la fisiología reproductiva ovina y la de otros rumiantes menores o mayores. Además, por varios años se la ha utilizado como modelo experimental para el estudio de la fisiología reproductiva humana ya que la hembra ovina comparte algunos aspectos endocrinoreproductivos con la mujer (Foster 1988).

La exposición prenatal a hormonas esteroidales como testosterona y cortisol ha permitido dilucidar aspectos claves de la fisiología ovina y su impacto en la hembra y macho adulto. Entre ellos, se han podido reconocer las consecuencias del estrés prenatal sobre la reproducción y el metabolismo del ovino adulto (De Blasio y col 2007) y últimamente se han podido establecer no sólo los aspectos organizacionales de los esteroides sobre la fisiología reproductiva de la hembra adulta sino que también sobre el macho (Recabarren y col 2006).

Aceptado: 19.11.2008.

\# Financiado por Proyecto Fondecyt 1050915.

* Casilla 537, Chillán, Chile; srecabar@udec.cl
Estudios pioneros en hembras ovinas prepúberes con exposición prenatal a testosterona (EPT) han podido establecer que la EPT durante el período crítico del desarrollo cerebral modifica el patrón de secreción de LH, conduciendo a un adelanto de la pubertad, lo cual a su vez depende del momento, duración y grado de la EPT (Kosut y col 1997). Este fenómeno, además, se hace independiente del fotoperíodo, lo cual es una característica de la activación del eje reproductivo del macho. Otros estudios han mostrado que la EPT ocasiona alteraciones en la morfología ovárica (Clarke y col 1977, Rojas-García y col 2006), en la conducta sexual y en la sensibilidad al retrocontrol de progesterona (Robinson y col 1999, Recabarren y col 2005').

Un aspecto que no se ha definido aún es si alguna de las consecuencias de la exposición prenatal a testosterona se puede reconocer al nacimiento, o bien sólo en etapas posteriores como la puberal o la adultez. Los estudios del grupo de Foster y de Padmanabhan (Wood y Foster 1998, Savabieasfahani y col 2005) han podido establecer que la EPT en altas dosis, sobre los $100 \mathrm{mg}$ de testosterona propionato, adelanta el inicio de la pubertad neuroendocrina y modifica el patrón de secreción de gonadotropinas del ciclo estral en hembras adultas. Además, estudios de nuestro laboratorio han demostrado que la EPT modifica la 
sensibilidad tisular a la insulina (Recabarren y col 2005a), la respuesta ovárica a la LH endógena en hembras ovinas inmaduras (Recabarren y col $2005^{\text {b }}$ ) y la secreción de LH y testosterona en respuesta al test de GnRH en machos maduros e inmaduros (Recabarren y col 2007). No obstante, aún no se han descrito parámetros biométricos y hormonales de machos y hembras recién nacidos con EPT. Es posible que la EPT modifique algunos parámetros biométricos y endocrinos que se pueden reconocer al nacimiento. En el presente trabajo se describen algunos de esos parámetros, entre los cuales se incluyen: hora del parto, número de crías, sexo de las crías, características morfológicas de los genitales, presencia de partos múltiples, peso al nacimiento, concentraciones plasmáticas de cortisol, como una medida del estrés del nacimiento y de las hormonas tiroideas como un indicador del estado metabólico.

\section{MATERIAL Y MÉTODOS}

\section{ANIMALES}

Para este estudio se utilizaron 60 hembras ovinas adultas de la raza Suffolk Down y sus respectivas crías (90 en total), nacidas durante tres períodos reproductivos. Las hembras se obtuvieron de un predio particular (Fundo Los Gultros, comuna de San Fabián de Alico, VIII Región).

A fines de marzo-principios de abril de cada año, las madres se sincronizaron y se aparearon con carneros de fertilidad probada. La sincronización de los estros, para monta dirigida, se logró con la aplicación de un implante vaginal de progesterona por 7 días (Eazy Breed ${ }^{\circledR}$, CIDR-G). Al momento de la remoción del implante, se inyectó prostaglandina F2- $\alpha$ (Genestren, Drug Pharma ${ }^{\circledR}$ ) intramuscular. Con este esquema de tratamiento se aseguró una fecha de cruzamiento y partos relativamente uniformes para la totalidad de las hembras.

Las hembras preñadas se distribuyeron al azar en tres grupos, un grupo control y dos de exposición prenatal a testosterona. Los protocolos de EPT de las madres se basaron según lo descrito por Kosut y col (1997) y Recabarren y col $\left(2005^{\mathrm{a}}\right)$. El primer protocolo utilizado consistió en la administración intramuscular de testosterona propionato (Steraloids Inc., USA) disuelta en aceite vegetal a un grupo de 16 hembras preñadas (EPT-P1) en concentraciones de 60 mg dos veces por semana, a partir de los 30 días y hasta los 90 días de gestación. En el segundo protocolo (EPTP2), a un grupo de 12 hembras preñadas se le administró $30 \mathrm{mg}$ de testosterona propionato intramuscular disuelta en aceite vegetal dos veces por semana, a partir de los 30 días y hasta los 90 días de gestación, dosis que posteriormente se elevó a $40 \mathrm{mg}$ de testosterona propionato desde los 90 hasta los 120 días de gestación. El grupo control, constituido por 32 hembras preñadas, se subdividió en dos grupos de 16 hembras cada uno, a las cuales sólo se les administró el vehículo consistente en aceite vegetal (30-90 días y 30-120 días).
Las ovejas preñadas se alimentaron con dieta estándar para hembras en gestación, en base a alimento pelletizado cuya composición consistía en materia seca (avena, maíz, trigo, harina de gluten, harina de soya, harina de pescado, harina de girasol y sales minerales) con $18 \%$ de proteína, $11 \%$ de fibra cruda, $2 \%$ de grasa y $2.450 \mathrm{Kcal} / \mathrm{kg}$ (Compañía Molinera El Globo, Chile), pradera natural y agua ad libitum. Además se mantuvieron bajo estricto control veterinario durante toda la gestación, con seguimiento ecográfico realizado por el Laboratorio de Reproducción Animal en la Facultad de Ciencias Veterinarias de la Universidad de Concepción en Chillán.

Las hembras parieron en los establos de la Facultad de Ciencias Veterinarias de la Universidad de Concepción, en un ambiente estabulado y constantemente controlado. Al momento del parto, los machos y hembras recién nacidos se dejaron sin intervención alguna durante ocho horas para permitir la vinculación madre-cría y asegurar la posterior ingesta de calostro durante las primeras horas de vida. Se registraron in situ la fecha y hora del parto, el tratamiento de la madre, el número de crías por madre, la condición general de la madre y su cría. Posteriormente, a las ocho horas postparto, se observó y registró el sexo de la cría y las características de los genitales externos y se midió la distancia ano-meato urinario o vagina con una huincha de medir. Además, se les registró el peso corporal mediante pesa digital y se les tomó la primera muestra de sangre por venopunción de la vena yugular. Además, como medida profiláctica, se limpió y desinfectó el cordón umbilical con una solución de polividona yodada al $10 \%$.

Las muestras de sangre se recibieron en tubos heparinizados (10 UI de heparina/1 $\mathrm{mL}$ de sangre). Los tubos se centrifugaron a $1.000 \mathrm{x} \mathrm{g}$ por 10 minutos a $4{ }^{\circ} \mathrm{C}$ (IEC Centra 7-R®). Posteriormente el plasma se rescató usando pipetas Pasteur y se depositó en microtubos Eppendorf ${ }^{\circledR}$ de 1,5 mL, los que se conservaron a $-20{ }^{\circ} \mathrm{C}$ hasta el momento de la determinación hormonal por radioinmunoanálisis (RIA).

\section{MEDICIÓN DE HORMONAS}

La medición de la concentración plasmática de cortisol, triyodotironina (T3) y de tiroxina (T4) plasmática se realizó en el Laboratorio de Fisiología y Endocrinología Animal de la Facultad de Ciencias Veterinarias (Universidad de Concepción) mediante RIA, utilizando kits comerciales (Coat-A-Count, DPC, USA). El RIA de cortisol presentó un coeficiente de variación intraensayo de $3 \%$ e interensayo de $7 \%$, y un nivel de detectabilidad de $5 \mu \mathrm{g} / \mathrm{dL}$ definido como el 90\% del buffer control (Recabarren y col 2000, Recabarren y col 2004). Para el RIA de T3, el coeficiente de variación intraensayo fue $2 \%$ e interensayo $5 \%$, y un nivel de detectabilidad de $13 \mathrm{ng} / \mathrm{dL}$ definido como el $90 \%$ del buffer control. Para el RIA de T4 los coeficientes intraensayo e intersayo fueron $2 \%$ y $5 \%$, respectivamente, y un nivel de detectabilidad de $0,97 \mu \mathrm{g} / \mathrm{dL}$ definido como el 90\% del buffer control. 
Todos los procedimientos experimentales realizados fueron aprobados previamente por el comité ético local para la investigación animal de la Facultad de Ciencias Veterinarias de la Universidad de Concepción.

\section{ANÁLISIS ESTADÍSTICO}

Se utilizó análisis de varianza (ANDEVA) de dos vías (dos sexos y tres tratamientos) con comparación de los promedios con el test de Newman Keuls para las variables hormonales y parámetros biométricos (distancia ano-meato urinario, pesos al nacimiento de las crías), utilizando el programa computacional GB-Stat v.5. Para cuantificar la relación entre las variables peso y concentración plasmática hormonal se utilizó la Correlación de Pearson a través del programa Prisma GraphPad 4,0. La hora del parto se distribuyó en cuatro períodos de seis horas cada uno, correspondiente a las 24 horas del día (24:00 a 05:59 h; 06:00 a 11:59 h; $12: 00$ a 17:59 h y 18:00 a 23:59 h). El tipo de parto y sexo de las crías se expresaron en forma porcentual. Se consideró para todos los análisis un $\mathrm{P}<0,05$ como estadísticamente significativo. Los datos se expresan como promedio \pm error estándar.

\section{RESULTADOS}

\section{PARÁMETROS BIOMÉTRICOS}

Horario de los partos. El cuadro 1 muestra la distribución porcentual de los partos en los cuatro períodos horarios descritos. Se registró un total de 86 crías (cuatro crías no fueron incluidas en este análisis porcentual por no tener el registro horario de su nacimiento).

En las madres control la mayor cantidad de nacimientos se concentró durante la noche en el período comprendido entre las 24:00 h y las 05:59 h (36\%), mientras que en las madres EPT-P1 la mayor cantidad de nacimientos se concentró en el período comprendido entre las 06:00 h hasta las 11:59 h (43,8\%). Finalmente, en las madres EPT-P2 la mayor cantidad de nacimientos se produjo durante el día y en dos períodos consecutivos, desde las 24:00 h hasta las 05:59 h (40\%) y desde las 06:00 h hasta las 11:59 hs (40\%).

Número de crías. El cuadro 2 muestra el porcentaje de partos que tuvieron como resultado una cría (parto único) o dos crías (partos melliceros). Considerando todos los partos, un 48,3\% fueron partos únicos y un 51,7\% fueron melliceros. Si se separa por grupo, las madres controles tuvieron mayormente partos únicos (parto único: 59,4\%; parto mellicero: $40,6 \%$ ), mientras que las madres EPT-P1 y EPT-P2 mayormente tuvieron parto mellicero (EPT-P1: parto único: 31,3\%; parto mellicero: 68,7\% y EPT-P2 parto único: 41,7\%; parto mellicero: $58,3 \%$ ).

El cuadro 2 muestra además para cada grupo de hembras el porcentaje de mellizos del mismo sexo y de sexos diferentes. En los partos en donde ambas crías fueron machos, el grupo EPT-P1 presentó la mayor cantidad con un 44,4\%; seguido por el grupo EPT-P2 con un $33,3 \%$ y el grupo control con un $22,2 \%$. El grupo de hembras control

Cuadro 1. Porcentaje de ovejas madres que paren en cuatro intervalos horarios durante las 24 horas del día. Las madres EPT recibieron testosterona propionato por 60 (P1) o 90 días (P2) a partir del día 30 de preñez mientras que las madres control recibieron aceite vegetal.

Percentage of ewes delivering during four intervals of time during a 24-hours day. EPT mothers received testosterone propionate for 60 or 90 days beginning at day 30 of pregnancy while control mothers received vegetable oil.

\begin{tabular}{lcccc}
\hline & \multicolumn{4}{c}{ Hora del día } \\
\cline { 2 - 5 } & $24: 00-05: 59$ & $06: 00-11: 59$ & $12: 00-17: 59$ & $18: 00-23: 59$ \\
\hline Madres control & $36 \%$ & $32 \%$ & $19 \%$ & $13 \%$ \\
Madres EPT-P1 & $25 \%$ & $43,8 \%$ & $2,5 \%$ & $18,8 \%$ \\
Madres EPT-P2 & $40 \%$ & $40 \%$ & $10 \%$ & $10 \%$ \\
\hline
\end{tabular}

Cuadro 2. Tipo de parto y sexo de crías melliceras nacidas de ovejas tratadas con testosterona durante la preñez y ovejas control. Las madres EPT recibieron testosterona propionato por 60 (P1) o 90 (P2) días a partir del día 30 de preñez mientras que las madres control recibieron aceite vegetal.

Delivery type and sex of the offspring born to ewes exposed to testosterone during pregnancy and to control sheep. EPT mothers received testosterone propionate for 60 or 90 days beginning at day 30 of pregnancy while control mothers received vegetable oil.

\begin{tabular}{lcccccc}
\hline & \multicolumn{2}{c}{ Tipo de parto } & & \multicolumn{3}{c}{ Sexo de los mellizos } \\
\cline { 2 - 3 } \cline { 6 - 7 } & Unico & Múltiple & & Macho-macho & Macho-hembra & Hembra-hembra \\
\hline Madres control & $59,4 \%$ & $40,62 \%$ & & $22,2 \%$ & $42,8 \%$ & $62,5 \%$ \\
Madres EPT-P1 & $31,3 \%$ & $68,7 \%$ & & $44,4 \%$ & $28,6 \%$ & $37,5 \%$ \\
Madres EPT-P2 & $41,7 \%$ & $58,3 \%$ & & $33,3 \%$ & $28,6 \%$ & $0 \%$ \\
\hline
\end{tabular}


presentó la mayor cantidad de partos con ambas crías hembras con un 62,5\%, el grupo EPT-P1 con un 37,5\% y el grupo EPT-P2 no tuvo este tipo de parto. Por último, en los partos en los que se obtuvo una cría de cada sexo el grupo de hembras control registró un 42,8\% y los grupos de hembras EPT-P1 y-P2 obtuvieron, ambos, un 28,6\%.

Sexo de las crías. En el cuadro 3 se entregan los porcentajes de machos y hembras nacidos en cada grupo de madres. Las hembras masculinizadas que poseían escroto vacío y pene fueron incluidas en el grupo de las hembras. En el grupo de las madres control, el mayor porcentaje de crías nacidas fue de sexo femenino con un $57 \%$; en el grupo de madres EPT-P1 el mayor porcentaje de crías nacidas fue de sexo masculino con un 59\%; y en el grupo de madres EPT-P2 el mayor porcentaje de crías nacidas fue de sexo masculino con un $63 \%$.

Peso de las crías. La figura 1 muestra la comparación entre los pesos al nacimiento de las crías nacidas de madres control, madres EPT-P1 y madres EPT-P2 en ambos sexos. Las hembras recién nacidas de madres EPT-P1 presentaron estadísticamente un menor peso al nacimiento en comparación a las hembras nacidas de madres control y nacidas de madres EPT-P2. Además pesaron menos que los machos de su mismo grupo y de los otros grupos $(\mathrm{P}<0,05)$.

Características de los genitales externos y distancia ano-meato urinario. Las hembras nacidas de madres EPT exhibieron una marcada masculinización de los genitales externos (figura 2), con la presencia de pene y un escroto vacío. Como complemento de la observación cualitativa se midió la distancia ano-meato urinario, la cual es un bioindicador de masculinización de la genitalia externa. En el cuadro 4 se muestra el promedio de las distancias ano-meato urinario separado por sexo y tratamiento. Las hembras EPT-P1 y EPT-P2 mostraron mayor distancia ano-meato urinario que las hembras control $(\mathrm{P}<0,01)$ y similar a la que presentaron los machos. La distancia ano-meato urinario de las hembras EPT-P2 fue menor a la de hembras EPT-P1, pero mayor que el de las hembras control $(\mathrm{P}<0,01)$.

Cuadro 3. Porcentaje de machos y hembras nacidos de ovejas expuestas a testosterona durante la preñez y de madres control. Las madres EPT recibieron testosterona propionato por 60 (P1) ó 90 (P2) días a partir del día 30 de preñez mientras que las madres control recibieron aceite vegetal.

Percentage of male and female lambs born to ewes exposed to testosterone during pregnancy and to control mothers. EPT mothers received testosterone propionate for 60 or 90 days beginning at day 30 of pregnancy while control mothers received vegetable oil.

\begin{tabular}{ccc}
\hline \multicolumn{1}{c}{ Grupos } & Machos & Hembras \\
\hline Madres control & $43 \%$ & $57 \%$ \\
Madres EPT-P1 & $59 \%$ & $41 \%$ \\
Madres EPT-P2 & $63 \%$ & $37 \%$ \\
\hline
\end{tabular}

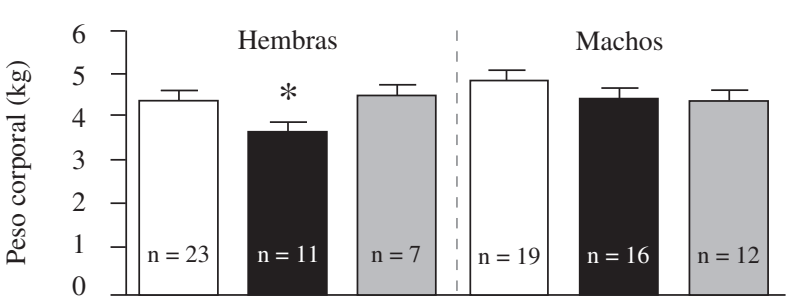

Figura 1. Peso corporal (promedio $\pm \mathrm{EE}$ ) en ovinos recién nacidos de madres control y de madres con exposición prenatal a testosterona. El color de la columna es indicativo del tratamiento (blanca $=$ control, negro $=$ EPT-P1, gris $=$ EPT-P2). Se muestran en forma separada hembras y machos. Dentro de cada columna se señala el número de recién nacidos de cada grupo. * indica diferencia significativa respecto a los otros grupo $(\mathrm{P} \leq 0,05$, ANDEVA).

Body weight (mean \pm SEM) in newborn lambs born to control mothers and to mothers with EPT. Colour of column indicates the treatment $($ white $=$ control, black $=$ EPT-P1, gray $=$ EPT-P2). Males and females are presented separately. Within each column, the number of newborn is given for each group. * indicates significant differences with respect to the other groups ( $\mathrm{P} \leq 0,05$, ANOVA).

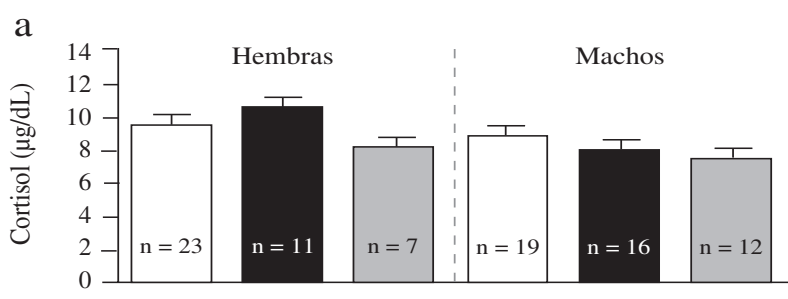

b

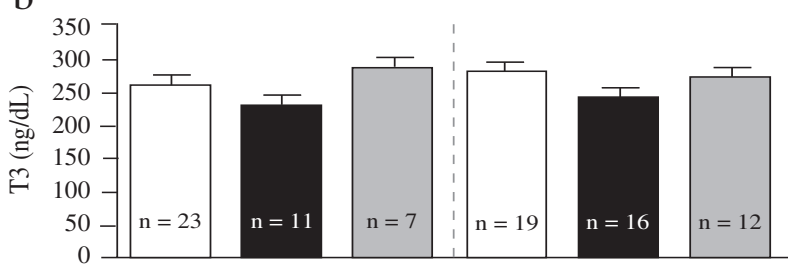

c

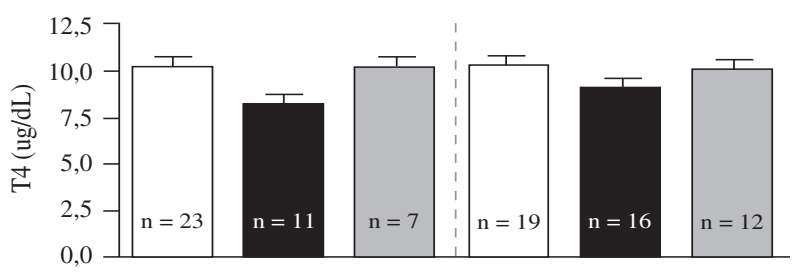

Figura 2. Concentraciones plasmáticas (promedio \pm EE) de a) cortisol, b) triyodotironina (T3) y c) tiroxina (T4) en ovinos recién nacidos de madres control y madres con EPT. El color de la columna es indicativo del tratamiento (blanca $=$ control, negro = EPT-P1, gris = EPT-P2). Se muestran en forma separada hembras y machos. Dentro de cada columna se señala el número de recién nacidos de cada grupo.

Plasma concentrations of a) cortisol, b) triiodothyronine (T3) and c) thyroxine (T4) in newborn lambs born to control mothers and to mothers with EPT. Colour of column indicates the treatment ( white $=$ control, black $=$ EPT-P1, gray $=$ EPT-P2). Males and females are presented separately. In each column, the number of newborns is given for each group. 
Cuadro 4. Distancia $(\mathrm{cm})$ ano-meato urinario en machos y hembras recién nacidos de ovejas expuestas a testosterona durante la preñez y de ovejas control. Las madres EPT recibieron testosterona propionato por 60 (P1) o 90 (P2) días a partir del día 30 de preñez mientras que las madres control recibieron aceite vegetal.

Distance from anus to urinary meatus in newborn male and female lambs born to ewes exposed to testosterone during pregnancy and to control mothers. EPT mothers received testosterone propionate for 60 or 90 days beginning at day 30 of pregnancy while control mothers received vegetable oil.

\begin{tabular}{lc}
\hline & Distancia $(\mathrm{cm})$ \\
\hline Machos control & $16,1 \pm 0,4^{\mathrm{a}}$ \\
Hembras control & $1,63 \pm 0,1^{\mathrm{b}}$ \\
Machos EPT-P1 & $15,4 \pm 0,45$ \\
Hembras EPT-P1 & $14,5 \pm 0,3$ \\
Machos EPT-P2 & $15,3 \pm 0,58$ \\
Hembras EPT-P2 & $11,5 \pm 2,0$ \\
\hline
\end{tabular}

A es distinto de $b(P \leq 0,05$, paired $t$ test $)$.

\section{PARÁMETROS ENDOCRINOS}

Concentraciones plasmáticas de cortisol, T3 y T4. La figura 2 muestra las concentraciones plasmáticas de cortisol, T3 y T4, en las crías macho y hembra nacidas de madres control y de madres EPT a las 8 horas postparto. No hubo diferencias estadísticas al comparar los tres grupos de crías. Las hembras nacidas EPT-P1 presentaron un promedio de cortisol plasmático de $8,99 \mu \mathrm{g} / \mathrm{dL}$, un valor levemente superior al de los otros grupos de hembras y machos (figura 2 a). Además, este grupo presentó un peso promedio significativamente más bajo. Es por ello que se evaluó la correlación entre ambos parámetros. Se encontró que en todas aquellas hembras nacidas EPT-P1 cuya concentración plasmática de cortisol fue mayor a $8,99 \mu \mathrm{g} / \mathrm{dL}$, el peso se correlacionaba inversamente con esta concentración de cortisol, es decir, aquellas hembras que tuvieron cortisol alto, su peso fue más bajo (figura 3). Esta correlación no se observó en los otros grupos de animales control o EPT-P2.

En relación a la T3, no hubo diferencias estadísticas al comparar los tres grupos de crías, mientras que en la T4 las hembras del grupo EPT-P1 mostraron una menor concentración de T4 que las hembras del grupo control. La correlación peso corporal-niveles de tiroxina es un importante bioindicador de estado metabólico en recién nacidos animales y humanos (De Blasio y col 2006, Fisher 2007). Al evaluar esa correlación en nuestros animales encontramos que en las hembras del grupo control existe una correlación directa entre peso corporal y T4 plasmática (figura 4). Esta correlación no está presente en machos y se pierde en las hembras nacidas EPT-P1 у EPT-P2.

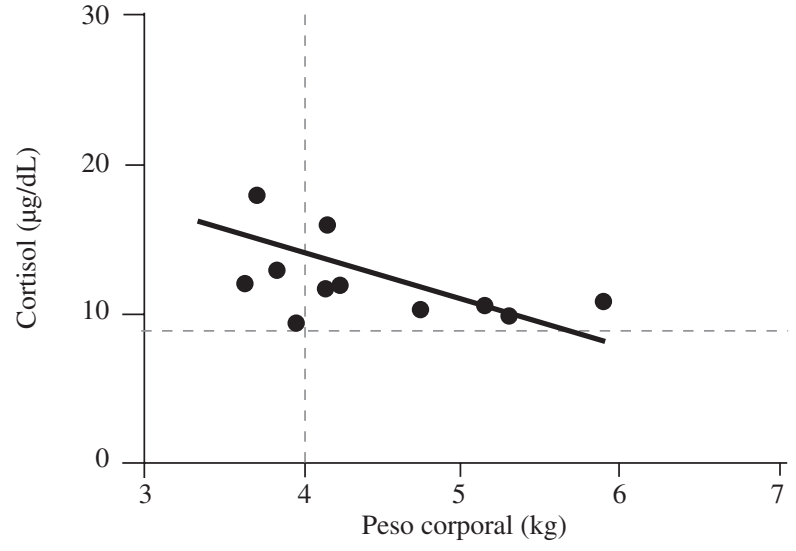

Figura 3. Correlación entre el peso corporal y el cortisol plasmático en corderos recién nacidos de madres EPT-P1. Cada punto en el gráfico indica un recién nacido. La correlación utilizada fue de Pearson, con un valor de $\mathrm{R}^{2}$ de 0,374 y un valor de $\mathrm{P} \leq 0,05$. La línea recta da cuenta del ajuste de los valores a la correlación. La pendiente de la línea recta es significativamente distinta de 0 .

Correlation between body weight and plasma cortisol concentrations in newborn lambs of mothers with EPT-P1. Each point in the figure indicates one newborn. Pearson correlation was used with a $\mathrm{R}^{2}$ of 0,374 and $\mathrm{P} \leq 0,05$. The slope of the straight line is significantly different from 0 .

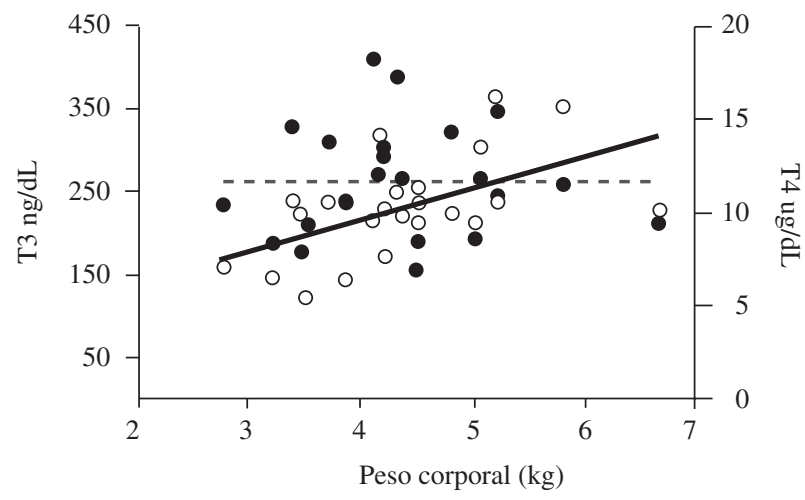

Figura 4. Correlación entre peso corporal y hormonas tiroideas en corderos hembras recién nacidos de madres control. Cada punto en el gráfico indica un recién nacido. La T3 está representada por los círculos negros y la T4 por los círculos blancos. La correlación utilizada fue de Pearson, con un valor de $\mathrm{R}^{2}$ de 0,302 y un valor de $\mathrm{P} \leq 0,05$. La línea recta da cuenta del ajuste de los valores a la correlación (línea punteada $=\mathrm{T} 3$, línea continua $=$ T4). La pendiente de la línea recta es significativamente distinta de 0 sólo para T4.

Correlation between body weight and T4 in newborn female lambs of control mothers. Each point in the figure represents one newborn lamb. Pearson correlation was used with a $\mathrm{R}^{2}$ of 0,302 and $\mathrm{P} \leq 0,05$. The slope is significantly different from 0 only for $\mathrm{T} 4$.

\section{DISCUSIÓN}

El presente estudio describe una serie de variables relacionadas con el parto de hembras ovinas Suffolk-Down y sus crías bajo condiciones de estabulación y medioambientales controladas y cómo puede una exposición 
prenatal a testosterona alterar estas variables. Las hembras con exposición prenatal fueron tratadas con testosterona propionato durante una etapa crítica del desarrollo del feto, lo que equivale a un cambio en el ambiente intrauterino que deja importantes efectos en el recién nacido. El mayor impacto en los parámetros analizados se observó en las hembras nacidas de madres EPT-P1 (60 mg de testosterona, dos veces por semana, del día 30 al 90 de gestación), las cuales, además de la masculinización de los genitales externos, exhibieron menor peso al nacimiento y menor concentración de tiroxina a las ocho horas postparto que las hembras control y machos. Las hembras nacidas de madres EPT-P2 también presentaron masculinización de los genitales externos, pero sin exhibir menor peso al nacimiento ni menor concentración de tiroxina que las control. Los machos nacidos de madres control y madres EPT no se diferenciaron entre sí en ninguno de los parámetros estudiados. Indudablemente, la aparición de aspectos fenotípicos masculinos en una hembra denota un fuerte efecto del tratamiento con testosterona sobre el desarrollo genital.

Los resultados en relación al parto muestran que los nacimientos en las hembras control se concentraron en dos intervalos horarios que abarcaron entre la medianoche y las seis de la mañana y entre las seis de la mañana y el mediodía. Cerca del 70\% de los partos ocurrieron entre esos horarios. El horario de inicio del parto podría estar en relación al ritmo circadiano de cortisol. El cortisol plasmático empieza a aumentar en las primeras horas de la madrugada en varias especies para alcanzar un máximo alrededor de las 07:00-08:00 h (Cagnacci y col 1998, Hernandes y col 2000). El ritmo circadiano de cortisol en los fetos previo al parto es desconocido, pero se ha evidenciado que los fetos de ovejas exhiben un ritmo de cortisol (Parraguez y col 1989). Lo observado en cuanto al horario de los partos es similar a lo encontrado en humanos. Estudios realizados en mujeres a punto de parir indican que existe una mayor incidencia de partos en la madrugada y al anochecer (Cagnacci y col 1998, Anderka y col 2000). Sin embargo, la distribución horaria de los partos en las madres EPT-P2 fue más tardía en comparación con las hembras control. La razón de ese retraso y desplazamiento desde las primeras horas de la mañana hasta el horario de la tarde es desconocida, pero podría estar relacionada con el ritmo circadiano de cortisol del feto. Cabe hacer notar que no se encontró una relación entre el sexo de la cría y la hora del parto.

En las hembras control, el 59,4\% de los partos fue parto único, mientras las hembras EPT fue mayormente de mellizos. La frecuencia de la obtención de mellizos es un carácter racial y, por tanto, de base genotípica, si bien se ha establecido que influyen en diversos grados factores ambientales, sobre todo la alimentación. No obstante, en los ovinos la heredabilidad (h2) de nacimientos gemelares es, en general, baja (10-15\%). Las madres de nuestro estudio eran todas multíparas. Si se toman en cuenta todas las madres, el porcentaje de madres melliceras sobrepasa levemente el 50\%. El tratamiento con testosterona a las madres no debería influir en el número de crías gestadas ya que la exposición a un exceso de testosterona se inicia a los 30 días postencaste, aunque Wolf y col (2002) observaron que en ratas la EPT puede llevar a efectos tóxicos con reducción en el tamaño de la camada y la viabilidad de los embriones. Además, el sexo de las crías nacidas de las hembras control es $57 \%$ hembras y $43 \%$ machos. Si se toma en consideración el total de crías, independiente del tipo de madre, el porcentaje de machos fue mayor que el de hembras ( $55 \%$ versus $45 \%$ ), producto de que las madres con los protocolos de exposición a testosterona parieron más machos que hembras. En este caso, se debe considerar que el tratamiento tampoco influiría en el sexo de la cría ya que éste se establece en el momento de la fecundación, a menos que la exposición prenatal a testosterona determinara una mayor mortalidad embrionaria de hembras a partir de los 30 días postgestación. Sin embargo, el seguimiento ecográfico no mostró signos de muerte embrionaria, por lo que los resultados en relación al sexo serían determinados por el azar.

Las hembras nacidas de madres EPT-P1 y -P2 presentaron masculinización de los genitales externos con la presencia de pene y un escroto vacío de menor tamaño que el de los machos y con una distancia ano-meato urinario semejante a los machos. Estos resultados, con dos protocolos diferentes de exposición prenatal a testosterona, confirman lo observado por otros autores. Se ha descrito que hembras nacidas de ovejas tratadas con $200 \mathrm{mg}$ de testosterona entre los 30 y 90 días de gestación nacen con genitales completamente virilizados, con un pseudopene y una bolsa escrotal vacía en lugar de una apertura vaginal (Herbosa y col 1995, Robinson y col 1999). Las hembras masculinizadas presentan ovarios y un útero con terminación ciega pero sin vagina (Unsworth y col 2005). Se ha establecido además, que el efecto virilizante observado en los genitales externos se acompaña de conductas masculinas en la hembra adulta (Orgeur 1995), de múltiples alteraciones neuroendocrinas y ováricas como el hipergonadotropismo (aumento en la secreción de hormona luteinizante, LH), la sensibilidad reducida al retrocontrol positivo y negativo de estradiol (Sharma y col 2002, Savabieasfahani y col 2005, Vergara 2007), el desarrollo de ovarios multifoliculares (Padmanabhan y col 2006, Rojas-García y col 2006), así como alteraciones en el perfil de LH y estradiol en la fase folicular del ciclo estral (Vergara 2007). En contraste, otros estudios han mostrado que las hembras nacidas de madres tratadas con testosterona entre los 60-90 días de gestación (segunda mitad del período crítico) nacen con genitales externos normales, pero tienen la postura urinaria masculinizada (Clarke y col 1976, Herbosa y col 1995), lo que permite concluir que el período crítico para la masculinización de los genitales externos ocurre entre los 30 y 60 días de gestación al igual que la masculinización del sistema nervioso central (SNC). 
Los efectos de la EPT en los genitales externos femeninos se han descrito en otras especies. En ratas, Wolf y col (2002) encontraron que la administración prenatal de propionato de testosterona condujo a la formación de genitales externos masculinos, con la distancia ano-genital aumentada. En humanos, las niñas expuestas prenatalmente a altos niveles de andrógenos, como en el caso de la hiperplasia suprarrenal congénita, presentan virilización de los genitales externos al nacimiento, incluyendo clitoromegalia y fusión de los labios (Nimkarn y New 2007, New 2004). El efecto de los andrógenos prenatales sobre el tracto reproductivo en la hembra se debería a que la testosterona y dihidrotestosterona mantienen el sistema de conductos de Wolff y promueven el crecimiento y desarrollo de glándulas accesorias y genitales externos de sexo masculino, mientras que la regresión del tracto reproductor femenino es principalmente dependiente de la hormona antimülleriana $(\mathrm{AMH})$ que se secreta desde la célula de Sertoli fetal (Josso y col 2006, Wolf y col 2002).

La exposición prenatal a testosterona influyó en el peso de nacimiento de las crías hembras de las madres EPT-P1. Las hembras recién nacidas EPT-P1 pesaron menos que las hembras control, que las hembras EPT-P2 y que los machos. Este fenómeno estaría asociado a la dosis y duración de la exposición a testosterona, dado que sólo se observa con el tratamiento EPT-P1. Estos resultados concuerdan con el estudio de Manikkam y col (2004) quienes observaron que el tratamiento con dosis de $100 \mathrm{mg}$ de testosterona propionato dos veces por semana entre los 30 y 90 días de gestación redujo el peso corporal y talla de los recién nacidos de ambos sexos y la circunferencia torácica de las hembras. En humanos, se ha propuesto que el peso al nacimiento es un indicador de la aparición de diferentes patologías en la edad adulta por los efectos "programadores" de esta condición (Eriksson y col 2001, Barker 2005, Barker y col 2006, Carlsen y col 2006). Resultados en recién nacidos humanos sugieren que la exposición prenatal a testosterona como aquella que se observa en madres con el síndrome de ovario poliquístico tiene como resultado recién nacidos con menor peso, lo que se conoce como niños pequeños para la edad gestacional (PEG) (Sir-Petermann y col 2002). Se ha atribuido también que el menor peso sería consecuencia de una alteración en la concentración de IGF-1 en el feto como resultado de la exposición a la testosterona (Padmanabhan y col 2006). Por otro lado, en el estudio de Dwyer y col (2006) se observó que el peso al nacimiento de los corderos estaba relacionado con el tamaño de la camada. Las gestaciones triples eran más cortas que las gestaciones únicas o gemelares. El peso al nacimiento de los corderos disminuía con cada aumento en el número de corderos en la camada. Sin embargo, en el presente estudio sólo las hembras EPT-P1 tuvieron un peso corporal menor al de los machos y hembras de otros grupos, pero este fenómeno no estaría relacionado con el tamaño de la camada. Los efectos de un bajo peso al nacimiento en ovinos se pueden evidenciar en sus desempeños productivos en la vida adulta, como, por ejemplo, reducción en el número de fibras musculares, lo que podría afectar el contenido y calidad de la carne (Fahey y col 2005). Sin embargo, otros estudios le atribuyen un efecto reductor en el contenido de grasa subcutánea o perirrenal o pélvica al tratamiento con testosterona prenatal, lo que podría contribuir a una mejor calidad de la carcasa en el animal al momento de su faena (DeHaan y col 1987).

En relación al cortisol plasmático, los resultados muestran que no hubo diferencia en los niveles plasmáticos al nacimiento entre los tres grupos. Estos resultados concuerdan con los de Manikkam y col (2004) quienes no encontraron diferencias significativas en los niveles del cortisol circulantes entre las borregas controles y las tratadas prenatalmente con testosterona. En el presente estudio no se observó una correlación entre peso al nacimiento y las concentraciones de cortisol, tomando en cuenta el total de recién nacidos o separados según el tratamiento de la madre. No obstante lo anterior, si se usa el valor de cortisol promedio de cada grupo como un límite de corte, se encontró una relación inversa entre peso al nacimiento y peso corporal en los recién nacidos EPT-P1. Esto ocurre sólo si se separa por cortisol y esta correlación no se repite en los recién nacidos de hembras control o nacidos de madres EPT-P2. En recién nacidos humanos se ha determinado que aquellos niños pequeños para la edad gestacional tienen mayor concentración de cortisol en la sangre del cordón umbilical que los niños adecuados para la edad gestacional (Economides y col 1988), lo cual sugiere que habría una relación entre el peso al nacimiento y las concentraciones de cortisol. Si se considera que el menor peso al nacimiento es el resultado final del crecimiento fetal en el útero, el cual depende principalmente de la disponibilidad de nutrientes, es posible suponer que hembras nacidas de madres EPT-P1 tuvieron menor disponibilidad de nutrientes que las hembras nacidas de hembras EPT-P2. Las razones de este fenómeno se desconocen, pero se ha demostrado en la oveja preñada que un aumento del cortisol induce una reducción en el número de células binucleadas de la placenta productoras del lactógeno placentario (Ward y col 2002), lo cual puede comprometer la disponibilidad del lactógeno placentario necesario para el crecimiento fetal por sus efectos en la partición de nutrientes y en las concentraciones de IGF-1 fetal (Braun y col 2007).

El cortisol es un factor relevante para la maduración fetal (Fowden y col 1998, Dwyer y col 2006) y para el aumento de la producción de triyodotironina (T3) en el feto. Esto último prepara al feto para el aumento en la demanda metabólica y la termogénesis que ocurren al nacimiento (Dwyer y col 2006). A su vez, las hormonas tiroideas promueven el desarrollo fetal y actúan como signos de disponibilidad de energía (Fowden y Forhead 2004). En la oveja, las hormonas tiroideas presentes en el plasma fetal derivan principalmente de fuentes fetales (Fowden y Forhead 2004). La triyodotironina se produce 
a partir de la tiroxina (T4) en el tejido adiposo pardo por la enzima 5'-monodeyodinasa, cuya actividad aumenta durante los últimos meses de preñez. Las concentraciones de T3 no se diferenciaron entre los grupos, mientras que la concentración plasmática de T4 fue menor en las hembras nacidas de madres EPT-P1. Mellor y Pearson (1977) describieron una menor concentración de $\mathrm{T} 4$ en recién nacidos durante las primeras 24 horas postparto con restricción del crecimiento fetal por disminución del tamaño de la placenta de las ovejas madres. En un estudio de Dwyer y col (2006), la T3 plasmática se relacionó significativamente con el peso al nacimiento durante las primeras 24 horas de vida, pero no después de esto. Los corderos de mayor peso tenían concentraciones plasmáticas mayores de T3 y T4 (Dwyer y col 2006). Esta asociación entre peso al nacimiento y T4 concuerda con nuestros datos. En primer lugar porque, a lo menos en las hembras, un alto valor de T4 coincidió con un peso mayor. Y en segundo lugar porque las hembras recién nacidas EPT-P1 presentaron una concentración promedio de T4 más baja que el resto de los grupos, y esas hembras tuvieron el menor peso promedio al nacimiento, aun cuando no encontramos una correlación directa entre ambas variables como en las hembras control. Las concentraciones de T3 y T4 fueron iguales entre los otros grupos ya que no diferían en el peso al nacer, lo que significaría que metabólicamente no habría diferencia entre los grupos.

Este estudio confirma la hipótesis planteada en relación a que las crías hembras expuestas prenatalmente a un exceso de testosterona durante su gestación exhiben diferencias en parámetros biométricos y endocrinos, tales como: distancia entre ano-meato urinario, peso al nacimiento y concentraciones de tiroxina (T4) en relación a las hembras nacidas de madres controles. En consecuencia, se puede inferir que el mayor impacto de la exposición prenatal a testosterona se produce en la hembra recién nacida. Las hembras presentan virilización de los genitales externos y dependiendo de la dosis y la duración de la EPT presentan menor peso al nacimiento y una menor concentración plasmática de T4. Sin embargo, en los otros parámetros biométricos y hormonales estudiados no se encontraron diferencias estadísticas entre crías de madres control y crías nacidas de madres expuestas a un exceso de testosterona, lo cual descarta efectos de la testosterona en la madre. Esto sugiere que el mayor impacto del exceso de testosterona en el feto y recién nacido se relaciona con la masculinización de las hembras. Aparentemente, la EPT no influiría en los parámetros estudiados en machos EPT, sin embargo, efectos de largo plazo no se pueden descartar.

\section{RESUMEN}

Se estudiaron parámetros biométricos y endocrinos de crías recién nacidas de ovejas Suffolk Down expuestas prenatalmente a un exceso de testosterona (EPT) durante 60 días (EPT-P1, $\mathrm{n}=16)$ y 90 días (EPT$\mathrm{P} 2, \mathrm{n}=12)$, a partir del día 30 de preñez. Las madres control $(\mathrm{n}=32)$ recibieron aceite vegetal. Al momento del parto se registraron: hora del parto, número de crías por madre y sexo de las crías. Ocho horas postparto se registraron: las características de los genitales externos, distancia ano-meato urinario (DAM) y peso corporal. Se determinó concentración plasmática de cortisol, T3 y T4, por radioinmunoensayo. Hora del parto, número y sexo de las crías fue similar entre los grupos. Las hembras nacidas de madres con EPT-P1 y EPT-P2 exhibieron genitales masculinizados con presencia de un pene y un escroto vacío. Las hembras nacidas de madres EPT-P1 presentaron menor peso al nacimiento que las hembras y machos control y hembras nacidas de madres EPT-P2 $(\mathrm{P}<0,05)$, DAM semejante a los machos $(\mathrm{P}<0,01)$ y menor concentración de $\mathrm{T} 4$ que los otros grupos $(\mathrm{P}<0,05)$. Además presentaron una relación inversa entre cortisol plasmático y peso corporal. Las hembras nacidas de madres EPT-P2, con excepción de la masculinización de los genitales, no se diferenciaron de las hembras nacidas de madres control. Los parámetros biométricos y endocrinos de los machos no mostraron diferencias entre los tres grupos. Concluimos que la EPT produce masculinización de los genitales de hembras y, dependiendo de la concentración de testosterona, la EPT puede disminuir las concentraciones plasmáticas de T4 en las hembras. La EPT no influye en parámetros biométricos y endocrinos de machos recién nacidos.

\section{AGRADECIMIENTOS}

Los autores expresan sus agradecimientos a los alumnos de pregrado de la carrera de Medicina Veterinaria de la Universidad de Concepción, Chillán, quienes colaboraron en la colección de los datos de los partos. Al Dr. José F. Cox y colaboradores del Laboratorio de Biotecnología de la Reproducción por el seguimiento ecográfico. Este trabajo fue financiado por Proyecto Fondecyt 1050915.

\section{REFERENCIAS}

Anderka M, ER Declercq, W Smith. 2000. A time to be born. Am J Public Health 90, 124-126.

Barker DJ. 2005. The developmental origins of insulin resistance. Horm Res 64, Suppl 3, 2-7.

Barker DJ, SP Bagby, MA Hanson. 2006. Mechanisms of disease: in utero programming in the pathogenesis of hypertension. Nat Clin Pract Nephrol 2, 700-707.

Braun T, S Li, TJ Moss, JP Newnham, JR Challis, PD Gluckman, DM Sloboda. 2007. Maternal betamethasone administration reduces binucleate cell number and placental lactogen in sheep. J Endocrinol 194, 337-347.

Cagnacci A, R Soldani, G Benedetto Melis, A Volpe. 1998. Diurnal rhythms of labor and delivery in women: modulation by parity and seasons. Am J Obstet Gynecol 178, 140-145.

Carlsen SM, G Jacobsen, P Romundstad. 2006. Maternal testosterone levels during pregnancy are associated with offspring size at birth. Eur J Endocrinol 155, 365-370.

Clarke IJ, RJ Scaramuzzi, RV Short. 1976. Effects of testosterone implants in pregnant ewes on their female offspring. J Embryol Exp Morphol 36, 87-99.

Clarke IJ, RJ Scaramuzzi, RV Short. 1977. Ovulation in prenatally androgenized ewes. J Endocrinol 73, 385-389.

De Blasio MJ, KL Gatford, JS Robinson, JA Owens. 2006. Placental restriction alters circulating thyroid hormone in the young lamb postnatally. Am J Physiol Regul Integr Comp Physiol 291, R1016-1024.

De Blasio MJ, M Dodic, AJ Jefferies, KM Moritz, EM Wintour, JA Owens. 2007. Maternal exposure to dexamethasone or cortisol in early pregnancy differentially alters insulin secretion and glucose homeostasis in adult male sheep offspring. Am J Physiol Endocrinol Metab 293, E75-E82.

DeHaan KC, LL Berger, DJ Kesler, FK McKeith, DL Thomas, TG Nash. 1987. Effect of prenatal androgenization on lamb performance, carcass composition and reproductive function. J Anim Sci 65, 1465-1470. 
Dwyer CM, CA Morgan. 2006. Maintenance of body temperature in the neonatal lamb: effects of breed, birth weight, and litter size. J Anim Sci 84, 1093-1101.

Economides DL, KH Nicolaides, EA Linton, LA Perry, T Chard. 1988. Plasma cortisol and adrenocorticotropin in appropriate and small for gestational age fetuses. Fetal Ther 3, 158-164.

Eriksson J, T Forsén, J Tuomilehto, C Osmond, D Barker. 2001. Size at birth, childhood growth and obesity in adult life. Int J Obes Relat Metab Disord 25, 735-740.

Fahey AJ, JM Brameld, T Parr, PJ Buttery. 2005. The effect of maternal undernutrition before muscle differentiation on the muscle fiber development of the newborn lamb. J Anim Sci 83, 2564-2571.

Fisher DA. 2007. Thyroid function and dysfunction in premature infants. Pediatr Endocrinol Rev 4, 317-328.

Foster DL. 1988. Chapter 40: Physiology of Reproduction. In: Knobil E, Neill JD (eds). Puberty in the female sheep. Raven Press, New York, USA, Pp 1739-1760.

Fowden AL, J Li, AJ Forhead. 1998. Glucocorticoids and the preparation for life after birth: are there long-term consequences of the life insurance. Proc Nutr Soc 57, 113-122.

Fowden AL, AJ Forhead. 2004. Endocrine mechanisms of intrauterine programming. Reproduction 127, 515-526.

Herbosa CG, RI Wood, DL Foster. 1995. Prenatal androgens modify the reproductive response to photoperiod in the developing sheep. Biol Reprod 52, 163-169.

Hernandes E, D Coelho, J Missel, D Kumpinski. 2000. Alteraciones circadianas del sistema cardiovascular. Rev Esp Cardiol 53, 117-122.

Josso N, JY Picard, R Rey, N di Clemente. 2006. Testicular anti-Müllerian hormone: history, genetics, regulation and clinical applications. Pediatr Endocrinol Rev 3, 347-358.

Kosut SS, RI Wood, C Herbosa-Encarnación, DL Foster. 1997. Prenatal androgens time neuroendocrine puberty in the sheep: effect of testosterone dose. Endocrinology 138, 1072-1077.

Manikkam M, EJ Crespi, DD Doop, C Herkimer, JS Lee, S Yu, MB Brown, DL Foster, V Padmanabhan. 2004. Fetal programming: prenatal testosterone excess leads to fetal growth retardation and postnatal catch-up growth in sheep. Endocrinol 145, 790-798

Mellor DJ, RA Pearson. 1977. Some changes in the composition of blood during the first 24 hours after birth in normal and growth retarded lambs. Ann Rech Vet 8, 460-467.

New MI. 2004. An update of congenital adrenal hyperplasia. Ann NY Acad Sci 1038, 14-43.

Nimkarn S, MI New. 2007. Prenatal diagnosis and treatment of congenital adrenal hyperplasia owing to 21-hydroxylase deficiency. Nat Clin Pract Endocrinol Metab 3, 405-413.

Orgeur P. 1995. Sexual play behavior in lambs androgenized in utero. Physiol Behav 57, 185-187.

Padmanabhan V, M Manikkan, S Recabarren, D Foster. 2006. Prenatal testosterone excess programs reproductive and metabolic dysfunction in the female. Moll Cell Endocrinolol 246, 165-174.

Parraguez VH, V Vergara, R Riquelme, R Arriman, AJ Llanos, M SerónFerré. 1989. Ontogeny of the circadian rhythm of cortisol in sheep. Biol Reprod 40, 1137-1143.

Recabarren SE, A Lobos, C Schneider, J Cox, J Parilo. 2000. Sensibilidad tisular a la insulina antes, durante y después de un ayuno en ovejas prepúberes. Arch Med Vet 32, 139-146.
Recabarren SE, A Lobos, V Torres, R Oyarzo, T Sir-Petermann. 2004. Secretory patterns of leptin and luteinizing hormone in food-restricted ewe lambs. Biol Res 37, 371-384.

Recabarren SE, V Padmanabhan, E Codner, A Lobos, C Durán, M Vidal, DL Foster, T Sir-Petermann. 2005 ${ }^{\mathrm{a}}$. Postnatal consequences of altered insulin sensitivity in female sheep treated prenatally with testosterone. Am J Physiol Endocrinol Metab 289, 801-806.

Recabarren SE, T Sir-Petermann, A Lobos, E Codner, PP Rojas-García, V Reyes. 2005 ${ }^{\mathrm{b}}$. Response to the gonadotropin releasing hormone agonist leuprolide in immature female sheep androgenized in utero. Biol Res 38, 235-244.

Recabarren SE, T Sir-Petermann, M Maliqueo, A Lobos, P Rojas-García. 2006. La exposición prenatal a andrógenos como factor de reprogramación fetal. Rev Med Chile 134, 101-108.

Recabarren SE, A Lobos, Y Figueroa, V Padmanabhan, DL Foster, T Sir-Petermann. 2007. Prenatal testosterone treatment alters LH and testosterone responsiveness to GnRH agonist in male sheep. Biol Res 40, 329-338.

Robinson JE, RA Forsdike, JA Taylor. 1999. In utero exposure of female lambs to testosterone reduces the sensivity of the gonadotropinreleasing hormone neuronal network to inhibition by progesterone. Endocrinology 140, 5797-5805.

Rojas-García PP, CI Abarca, CE Ulloa, V Alfaro, MP Recabarren, DA Sandoval, T Sir-Petermann, SE Recabarren . 2006. Estudio preliminar de la histología ovárica en ovejas adultas con exposición prenatal a testosterona (EPT). XVII Reunión Anual Sociedad Chilena de Reproducción y Desarrollo, Reñaca, Chile.

Savabieasfahani M, JS Lee, C Herkimer, TP Sharma, DL Foster, V Padmanabhan. 2005. Fetal programming: testosterone exposure of the female sheep during midgestation disrupts the dynamics of its adult gonadotropin secretion during the periovulatory period. Biol Reprod 72, 221-229.

Sharma TP, C Herkimer, C West, W Ye, R Birch, JE Robinson, DL Foster, V Padmanabhan. 2002. Fetal programming: prenatal androgen disrupts positive feedback actions of estradiol but does not affect timing of puberty in female sheep. Biol Reprod 66, 924-933.

Sir-Petermann T, M Maliqueo, B Angel, HE Lara, F Pérez-Bravo, SE Recabarren. 2002. Maternal serum androgens in pregnant women with polycystic ovarian syndrome: possible implications in prenatal androgenization. Hum Reprod 17, 2573-2579.

Unsworth WP, JA Taylor, JE Robinson. 2005. Prenatal programming of reproductive neuroendocrine function: the effect of prenatal androgens on the development of estrogen positive feedback and ovarian cycles in the ewe. Biol Reprod 72, 619-627.

Vergara F. 2007. Caracterización de los perfiles de LH, Estradiol y Progesterona del ciclo estral durante la segunda estación reproductiva en ovejas Suffolk con exposición prenatal a testosterona. Memoria de título, Facultad de Medicina Veterinaria, Universidad de Concepción, Chillán, Chile.

Ward JW, FB Wooding, AL Fowden. 2002. The effects of cortisol on the binucleate cell population in the ovine placenta during late gestation. Placenta 23, 451-458.

Wolf CJ, A Hotchkiss, JS Osthy, GA LeBlanc, LE Gray Jr. 2002. Effects of prenatal testosterone propionate on the sexual development of male and female rats: a dose-response study. Toxicol Sci 65, 71-86.

Wood RI, DL Foster. 1998. Sexual differentiation of reproductive neuroendocrine function in sheep. Rev. Reprod 3, 130-140. 
